\title{
Abstract: Automatic Malignancy Estimation for Pulmonary Nodules from CT Images
}

\author{
Katrin Mentl, Rimon Saffoury, Andreas Maier \\ Pattern Recognition Lab, Friedrich-Alexander University of Erlangen-NÃ $\frac{1}{4} \mathrm{rnberg}$ \\ katrin.mentl@fau.de
}

Early detection of lung cancer is crucial to increase the chance of cure. As lung cancer often manifests itself in the presence of malignant pulmonary nodules, the assessment of such is of high clinical importance. Lung cancer screening is primarily performed using diagnostic imaging modalities such as CT, while invasive methods such as biopsy are used as a last resort to confirm diagnosis.

Various prediction models that are based on both patient (e.g., age, smoking status) and radiological features (e.g., nodule's type) have been suggested. However, the manual extraction of the essential radiological characteristics from CT scans is a cumbersome and time-consuming task, which is, moreover, subject to inter-observer variation.

To overcome these limitations, we propose a pipeline which automatically extracts relevant features from a CT baseline scan and outputs a malignancy probability score based on established prediction models such as the Mayo Clinic model. The following radiological features are extracted: presence of emphysema, nodule's diameter, nodule's type and presence of spiculation. The presence of emphysema is classified based on the ratio of low attenuation areas to the lung volume, while the other features mainly depend on the automatic lung nodule segmentation. The nodule type, i.e. solid, part solid or non-solid is determined using a two-step classification scheme and the presence of spiculation is detected with a SVM classifier.

We evaluated our segmentation approach on over 1,000 nodules and reached a low mean absolute error of around $1 \mathrm{~mm}$. We achieved high accuracies across all classification tasks and promising performance on the investigated prediction models. Thus, we propose to consider the system as decision support in routine clinical practice. 